\title{
Sécurité des patients en médecine de premier recours - premières données suisses
}

Katrin Gehring,

David Schwappach

Correspondance:

Dr Katrin Gehring

Fondation pour la Sécurité des

Patients

Asylstrasse 77

CH-8032 Zurich

gehring@patientensicherheit.ch

\begin{abstract}
La médecine de premier recours - longtemps «parent pauvre» de la sécurité des patients

Alors que la majorité des contacts avec les patients ont lieu en médecine de premier recours, le mouvement pour la sécurité des patients a concentré son action pendant plusieurs années essentiellement sur les hôpitaux. Or nombre d'études réalisées dans le monde entier montrent que beaucoup d'erreurs et d'incidents se produisent aussi en médecine ambulatoire. Les soins dispensés dans ce domaine étant soumis à des organisations très différentes d'un pays à un autre, il est difficile d'appliquer les résultats de ces études à notre pays. Par ailleurs, la médecine de premier recours se trouve dans une phase de mutation, aussi bien en Suisse que dans de nombreux pays. Les nouvelles formes d'organisation des soins, à l'instar du Managed care ou de la création d'immenses cabinets de groupe, assimilables à de grands centres de soins, deviennent ainsi les nouveaux enjeux de la sécurité des patients.
\end{abstract}

\section{Etude de la Fondation pour la Sécurité des Patients}

Grâce à cette première collecte de données concernant la sécurité des patients traités en médecine de premier recours, la Fondation comble cette lacune. Les personnes interrogées, des médecins et des assistantes médicales exerçant dans des cabinets suisses alémaniques, toutes affiliées à un réseau de médecins (13 réseaux appartenant à 4 organisations principales), ont répondu par écrit à un questionnaire standardisé. En plus des raisons pragmatiques (accessibilité, ressources), l'étude se concentre sur des cabinets organisés en réseau parce qu'il est probable que les réseaux de soins deviennent une forme d'organisation qui sera de plus en plus courante dans le secteur ambulatoire.

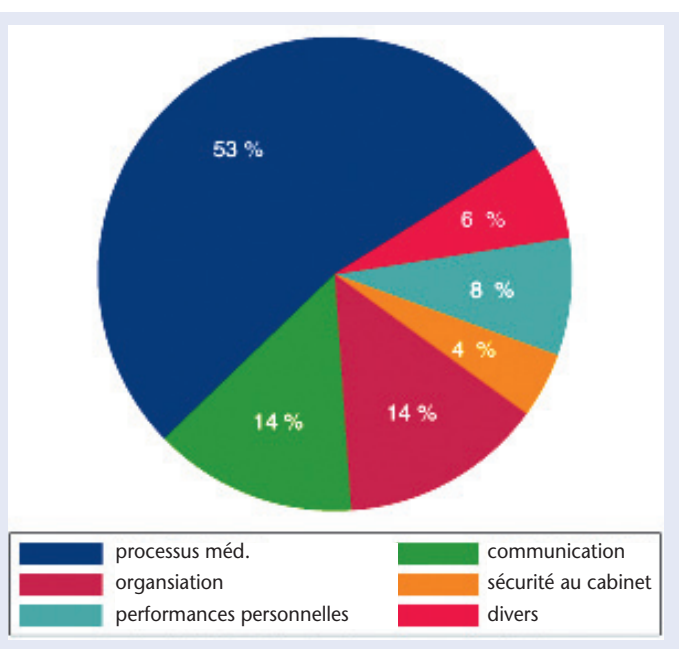

performances personnelles
L'enquête a recensé les événements critiques qui surviennent au cabinet et les a analysés d'après leur fréquence dans un environnement de travail donné et selon l'ampleur des dommages occasionnés. Cela a permis de les classer dans les catégories suivantes: processus diagnostique, médication, autres mesures préventives et thérapeutiques, information au et contact avec le patient, organisation et processus au cabinet, collaboration avec les partenaires externes ainsi que médicaments et matériel.

Variable systémique liée à l'environnement du cabinet, le climat de sécurité dans les cabinets de médecins de famille est susceptible d'influencer le risque d'erreurs et de provoquer des événements indésirables. L'étude l'a donc également passé au crible. La mise en œuvre réussie de mesures pour améliorer la sécurité des patients présuppose un climat de sécurité positif, dont les principaux aspects se déclinent en travail d'équipe, satisfaction professionnelle et charge de travail, mode de direction, mesures pour éviter les erreurs et gestion des erreurs.

L'étude a accordé une valeur particulière au fait que la sécurité des patients est étroitement subordonnée à la communication entre les groupes professionnels, aux questions à la base des interactions professionelles et au travail d'équipe. En plus de l'appréciation et de l'évaluation par des médecins des problèmes de sécurité et du climat de sécurité, l'étude a également réalisé une analyse systématique du point de vue des assistantes médicales dans les cabinets de médecine de premier recours; elle a ainsi pu mettre en évidence les différences existant entre ces deux groupes professionnels et fournir des informations sur un domaine relativement peu connu jusqu'à présent.

\section{Résultats}

Sur l'ensemble des personnes contactées, 633 ont répondu au questionnaire. Le taux de participation s'élève ainsi à 50\% aussi bien chez les médecins que les assistantes médicales. Les résultats montrent que les événements critiques pris en compte dans l'étude ne représentent aucunement des cas isolés: sur les 23 événements critiques analysés, $30 \%$ des médecins et $17 \%$ des assistantes médicales mentionnent en rencontrer au moins un par jour ou par semaine.

$12,6 \%$ de l'échantillon indique s'être trouvé tous les jours ou toutes les semaines face à des dossiers de patients incomplets ou criblés d'erreurs au cours des 12 derniers mois. La dernière fois qu'une erreur diagnostique a été commise ou qu'un diagnostic a été posé trop tard au cabinet, 4,8\% des personnes interrogées évoquent des conséquences considérables pour le

\section{Figure 1}

Ensemble des risques indiqués par les soignants à traiter en priorité au cabinet médical.

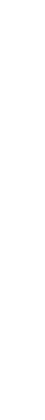

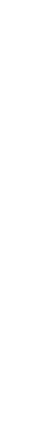

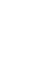

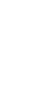


patient, qui ont conduit à sa mort ou à des lésions graves.

Les participants ont nommé 936 risques qu'ils «neutraliseraient» en priorité dans leur cabinet, si c'était possible.

Dans cette énumération, 53\% concernent les risques relatifs aux processus médicaux, dont en particulier la médication (28\%), les actes réalisés (p. ex. vaccins, radios) au cabinet (11\%), le triage (7\%) et le diagnostic (5\%). Les autres réponses se concentrent sur les risques lors de la communication, aussi bien en interne mais aussi avec des partenaires externes (14\%), l'organisation (14\%), la sécurité au cabinet (par ex. chutes, risques d'infection) (4\%) et les performances personnelles (par ex. fatigue, erreur due à la mémoire) (8\%).

Sur la question du climat de sécurité, l'étude a mis en évidence des différences significatives entre les groupes professionnels: les assistantes médicales par exemple attribuent une note beaucoup moins bonne que les médecins à l'entraînement régulier pour les situations d'urgence les plus courantes. Les médecins ont également une meilleure appréciation des discussions à propos des erreurs au sein de l'équipe du cabinet.

\section{Conclusion}

Les résultats permettent de déduire des champs d'action concrets: par exemple le triage des appels téléphoniques des patients est un domaine à risque, qui pose problème à un grand nombre des assistantes médicales mais aussi des médecins interrogés. Dans le même temps, l'étude relève que le point critique «Urgence non reconnue d'une demande de patient» est considéré comme particulièrement important en raison des conséquences graves qu'il peut provoquer. Deux autres domaines représentent un terrain à risque sur lequel il est important d'agir, à savoir la «médication» avec toutes ses facettes mais aussi la collaboration avec des organismes/partenaires externes (par ex. hôpitaux, spécialistes). Ces conclusions permettent de cibler des activités pour la sécurité des patients qui reflètent la situation concrète dans les cabinets et se basent sur l'expertise mais aussi sur les besoins des praticiens. Grâce à la présente étude, nous disposons, pour la première fois et pour un échantillon relativement important, de données concernant le climat de sécurité et les risques concrets en matière de sécurité des patients dans les cabinets de médecine de premier recours suisses. La participation relativement élevée et homogène des médecins et des assistantes médicales sollicités montre que la sécurité des patients est un sujet essentiel pris très au sérieux dans les cabinets médicaux. Conjointement avec des partenaires exerçant dans ce domaine, la Fondation pour la Sécurité des Patients développera des offres en vue d'améliorer la sécurité des patients en médecine de premier recours.

\section{Remerciements}

Nous adressons tous nos remerciements aux réseaux de médecins eastcare (Dr Roman Buff, Peter Sauter), hawadoc (Dr Markus Wieser), mediX bern (Dr Markus Battaglia) et mediX zürich (Dr Felix Huber) pour leur collaboration engagée dans cette étude. Nous remercions aussi particulièrement tous les médecins et toutes les assistantes médicales qui ont répondu au questionnaire ainsi que les Drs Andreas Brun et Kurt Hess pour leur soutien et leur expertise.

L'étude «Safety Hot-Spots und Sicherheitsklima in Schweizer Praxen der Grundversorgung» a été cofinancé par la FMH.

Le projet a remporté le prix 2011 du Forum Managed Care.

Lors du congrès international de la Fondation pour la sécurité des patients, la question de la sécurité des patients dans la médecine de premier recours sera traitée dans une des nombreuses sessions parallèles:

Sécurité des patients en médecine de premier recours - avanti!

Au début du $3^{\mathbf{e}}$ millénaire: quels progrès, quelles perspectives?

\section{9/30 novembre 2011}

Pré-conférence 28 novembre 2011, Congress Center Basel

\section{Des pionniers de premier plan et de grands experts internationaux}

Charles Vincent (Imperial College de Londres) et Robert Wachter (Université de Californie à San Francisco) figurent parmi les meilleurs chercheurs et experts au monde dans le domaine de la gestion des risques cliniques et de la gestion d'entreprise axée sur la sécurité. Kathleen Sutcliffe (Université du Michigan) est l'une des plus grandes spécialistes mondiales en matière d'organisation et de culture dans les industries à haut risque. Martin Bromiley a été pilote et «Human Factor Trainer». Depuis la perte de son épouse en 2005 suite à une erreur médicale, il lutte au sein du système de santé britannique pour que les facteurs humains deviennent un élément clé de la prévention des erreurs. Le programme sera également marqué par la présence d'excellents chercheurs et praticiens venus de Suisse et des pays voisins.

Les principaux sujets abordés seront les suivants: la sécurité des patients - hier et demain; gestion, stratégie et bénéfice pour les entreprises, facteurs humains, sécurité maximale, sécurité et implication des patients, questions juridiques. Par ailleurs, des sessions parallèles avec de brefs exposés et des ateliers interactifs permettront d'approfondir beaucoup d'autres sujets. Une exposition de posters avec un concours offrira également la possibilité d'échanger sur les projets et les activités sur la sécurité des patients. Pour de plus amples informations: www.patientensicherheit.ch/kongress 